\title{
EL OBJETO Y LA CONSTRUCCIÓN DE SENTIDO EN COLECCIONES ETNOGRÁFICAS
}

\author{
María Marta Reca*
}

\begin{abstract}
RECA, M.M. El objeto y la construcción de sentido en colecciones etnográficas. Rev. do Museu de Arqueologia e Etnologia, São Paulo, 6: 269-273, 1996.
\end{abstract}

RESUMO: As condições de análise da cultura material e os marcos cenceituais, a partir dos quais podem ser lidos os objetos, adotam um caráter singular no estudo de coleções etnográficas. A partir da apresentação des aspectos teórico-metodológicos vinculados ao tipo de referente tratado e aos processos de interrogação a que são submetidos os «objetos» pelo investigador, se propõem diversos níveis de contextualização que põem em evidência a passagem da dimensão material à dimensão simbólica, constituindo as instâncias referenciais de atribuição de sentido. Este processo se exemplifica em um conjunto de máscaras que fazem parte do Departamento Científico de Etnografia do Museu de La Plata.

UNITERMOS: Cultura material - Coleção - Etnografia - Contexto de significação - Estudo descritivo.

\section{Introducción}

El objetivo del presente trabajo es el de exponer un conjunto de consideraciones en torno al análisis de la cultura material y sus posibilidades de interpretación. Las mismas surjen del estudio de colecciones del Departamento Científico de Etnografía del Museo de La Plata.

El estudio de colecciones etnográficas constituye un área de investigación que generalmente es dejado para los museólogos o descuidado por los etnógrafos que encuentran en la inmediatez del trabajo de campo el modo de aprehensión de los contextos de significación en los que se inscribe un objeto. Este campo de estudio presenta condiciones particulares y es propicio para la reflexión teórica, y permite por un lado, la aproximación de la práctica museológica al conocimiento antropoló-

(*) Departamento Científico de Etnografía del Museo de Ciencias Naturales de La Plata. U.N.L.P. gico, por otro, indagar acerca de las condiciones de representatividad que el objeto encierra en su materialidad.

Un objeto cualquiera puede ser considerado como etnográfico en la medida en que es posible reconocer un contexto de recolección que se genera a partir de la presencia del etnógrafo en un grupo particular y del cual el objeto es extraído para formar parte de una colección.

Así, a diferencia del objeto arqueológico, cuyo contexto de recolección sin sujeto, está compuesto por las asociaciones materiales en terreno, el objeto etnográfico ocupa un lugar entre otro conjunto de documentos tales como textos etnográficos, libretas de campo, registros fílmicos y fotográficos, por medio de los cuales es posible recomponer sus usos y significados en una cultura en particular.

Esta aparente «ventaja» del segundo respecto del primero, se vuelve paradójicamente negativa si pensamos que ha llevado, en muchos casos, a restringir el estudio de colecciones a una práctica de adjudicación de sentido basada en la correspon- 
dencia del discurso etnográfico con el objeto aludido, proceso de reconstrucción de un contexto en el que el investigador queda fuera de la secuencia interpretativa. No es la intensión minimizar el valor de la documentación etnográfica, por el contrario, supone un observador activo e involucrado en la valorización del objeto.

Recientes estudios han desmistificado el trabajo de campo, cuestionando las condiciones en las cuales es posible generar un conocimiento acerca de otra cultura (Marcus 1991). Estos análisis críticos, de los que no nos ocuparemos aquí, han llevado al reconocimiento, en mayor o menor medida, de la intervención del antropólogo observador en la secuencia explicativa del fenómeno observado, intervención que ya no queda resguardada en la etnografía por la presencia de el "otro" y que, en todo caso hay que explicitar en los procesos de construcción de sentido.

En el tratamiento de la cultura material, la posibilidad de su manipulación, la cuantificación de sus caracteres, la perdurabilidad de sus condiciones, hacen que se constituya como evidencia. Sin embargo, todo objeto podrá ser interpretado sólo si se lo incluye en un contexto, ya que "...los valores no están en los objetos sino que dependen de las valoraciones que de ellos hacemos."(Mathieu 1987).

Es así que un mismo objeto admite distintas lecturas según el marco de referencia elegido para desbordar los límites de su materialidad e ingresar en un campo más difuso e interpretativo.

\section{Corpus de referencia}

Trataremos de ejemplificar algunos de los niveles de contextualización de los objetos a través de un corpus constituído por un conjunto de más de cien máscaras que forman parte de las colecciones del Departamento de Etnografía. Las mismas fueron recolectadas por el Dr. Enrique Palavecino entre los grupos chiriguano chané de la región chaqueña argentina, en los años 1947- 49. Esta colección presenta condiciones favorables para su análisis, ya que además de contar con un número significativo de ejemplares, se encuentra en buen estado de conservación, y acompañada de publicaciones basadas en información de campo (Palavecino1954, Roca1972).

A estas consideraciones museológicas se suman otras que abren múltiples vías de acceso en el análsis, a saber:
1) Universalidad: la máscara aparece como u elemento que, asociado al ritual, la muerte, 1 guerra, etc. ha sido registrada en la mayoría di las culturas, tanto del pasado como en el presen te, en sociedades simples y complejas, sobr todo si pensamos que dentro de este concept se incluyen diversas modalidades de enmas. caramiento tales como pinturas corporales, tatuajes, figuras de mascarillas grabadas en otros objetos como bastones de mando, etc. Es decir que todas las culturas encontramos la presencia, explícita o no, de este tipo de elemento.

2) Diversidad de rasgos: la máscara es un tipo de objeto que presenta una gran riqueza en la combinación de formas, colores, diseños y elementos decorativos. Esta característica permite la construcción de un sistema complejo de combinación de rasgos que definen diversas posibilidades estilísticas. Ella puede ser considerada una obra de arte aludiendo a la creatividad del artesano, y por lo tanto presentar un alto grado de ambiguedad. “...las máscaras deben ser creaciones de la imaginación humana $y$, en consecuencia, ambiguas. De hecho demuestran ambiguedad por su propia estructura." (Tonkin 1979).

3) Práctica actual: la práctica actual de la confección de máscaras por parte de los grupos chané y la vigencia de su uso en el contexto del carnaval, permite abordar estudios comparativos en un eje diacrónico, de manera de evaluar las transformaciones tecnológicas y sus posibles causas. Estos grupos desarrollan una verdadera artesanía de la máscara distinguiendo, en la actualidad, aquellas que responden a una tradición y que son confeccionadas para su uso en el evento del carnaval, y aquellas que son confeccionadas para la venta y que, en consecuencia pierden su valor ritual para adquirir un valor económico. Este cambio de valor se traduce en la materialidad de la máscara que, si bien conserva ciertos rasgos identificatorios de esta cultura en particular, incorpora nuevos elementos y combinación de formas.

\section{Metodologia}

En una primera aproximación al análisis descriptivo de los referentes, se practicaron los lineamientos generales del método analítico-descriptivo 
definido por J.C. Gardin (1975) para el tratamiento de los materiales arqueológicos. Este autor expone un conjunto de reglas que permiten orientar, segmentar y diferenciar la información perceptual de manera de elaborar una base de datos firme para posteriores interpretaciones. A partir de la descripción del referente, se genera un conjunto de datos primarios bajo la condición de explicitar los criterios desde los cuales un elemento es distinguido como significativo. Se busca estandarizar las descripciones a través de la construcción de un lenguaje descriptivo entendido como un sistema de reescritura o código desde el cual es posible recomponer la unidad de referencia. En un principio se asumió este método en todas sus dimensiones, diluyendo los aspectos diferenciales de los materiales arqueológicos y etnográficos. Sin embargo, las dificultades surgidas en el análisis llevaron a un ajuste de los procedimientos metodológicos, atendiendo principalmente a la heterogeneidad con que se nos presentan las máscaras y a la ambiguedad propia del acto creativo.

Así, fueron definidos los planos de orientación privilegiando aquel que, por analogía con el rostro, presenta la mayor cantidad de elementos tales como ojos, nariz, boca, mentón, etc. Allí se "concentra" la mayor cantidad de elementos que combinados otorgan a la máscara su caracter representativo. A su vez, esta cara fue segmentada en una serie de regiones según la presencia y/o ausencia de estos elementos, en cada una de las cuales fue posible describir una serie de variables tales como materia prima, forma, dimensiones, técnica, emplazamiento, entre otros. Cada una de estas variables asume distintos valores hasta llegar a componer un grilla de alternativas y sus posibles relaciones.

De igual manera, respetando la orientación y segmentación para la morfología, se describieron los elementos decorativos u ornamentales, definidos como aquellos elementos agregados que se suman a la conformación del rostro tipo. Así, por ejemplo se consideró un nuevo nivel de emplazamiento (por encima de, por debajo de, sobre, entre, etc.), al que se acopla el tipo y modo de elemento agregado. Por ejemplo, incrustaciones de plumas en el contorno del rostro.

El objeto, sometido a este proceso de descontextualización en el que se definen las unidades descriptivas, podrá ser recompuesto desde el sistema descriptivo construido, el cual deberá incluir los criterios, los niveles lógicos y las categori- zaciones. En principio cualquier componente aislado puede constituir un rasgo descriptivo, sin embargo, es en la definición de parámetros, en un juego de ida y vuelta desde el objeto al criterio y viceversa, que es posible definir los Niveles de comparabilidad en que el rasgo adquiere Capacidad descriptiva dentro de un corpus de objetos dados. De esta manera se instaura un proceso de jerarquización del rasgo discriminando:

a) aquellos rasgos con capacidad descriptiva en la totalidad del corpus y cuya relación define el tipo de referente. Consiste en el conjunto de rasgos que responden a los requisitos morfológicos por los cuales un elemento es considerado una máscara y distinguido de otra serie de objetos. Por ejemplo, que este permita cubrir parcial o totalmente el rostro, que pueda ser portada durante cierto tiempo, etc.

b) aquellos rasgos compartidos por un subtipo y cuya relación define una identidad de clase. Es decir, características morfológicas diferenciales que definen, por ejemplo, tendencias estilísticas que, en el caso estudiado, responden a la identificación de los seres que representan y al momento del día en que son usadas.

c) aquellos rasgos que definen un único objeto y cuya relación le otorga identidad individual.

\section{Contextos de significación}

A partir de aquí es posible sistematizar las particularidades formales del objeto y retener las similitudes y diferencias de un objeto a otro, pudiendo ser reconocidos aquellos elementos y sus modos de combinación que le otorgan a cada una su carácter representativo. Es decir, permite identificar los sistemas relacionales que dentro de una gran variedad de combinatorias, mantiene ciertas constantes en su articulación, poniendo de manifiesto los elementos estructurales.

Llamaremos a este primer nivel de análisis, contexto analítico-descriptivo, concebido como una red de relaciones formales. Un segundo nivel o contexto funcional, que alude al conjunto de acciones y actitudes, circunstancias de uso, confección, participantes, que reflejan una práctica social. Permite leer la correspondencia entre la forma, la materia y la función específica que le es culturalmente asignada al objeto. 
Por último, un contexto simbólico que refiere a su carácter representativo. La máscara, entre los chané, constituye el elemento mediatizador entre el mundo de los vivos y el mundo de los muertos, el puente entre el mundo natural y el mundo sobrenatural a través de la identificación en ella de los espíritus de los muertos. El portador de una máscara adquiere una nueva identidad, con carácter transitorio y desarrolla un comportamiento acorde al personaje que representa. Así, las máscaras pueden ser interpretadas como operadores en eventos comunicativos, e interrogarnos acerca de cuáles son los requisitos formales que debe cumplir para transmitir el mensaje adecuado.

\section{Comentarios finales}

De qué manera la información acerca de una cultura se encuentra contenida en la materia? Qué conjunto de relaciones contextuales permiten trascenderla y abordar sus significados?
Los objetos pueden ser concebidos como elementos portadores de valores culturales. Sabemos que antes de ser costruído, el objeto fue pensado la técnica, antes de ser utilizada, fue aprendida; la materia, antes de ser modelada, fue adquirida, fue concebida como posibilidad. Su análisis devela aspectos multifacéticos y sus mensajes podrán ser decodificados en la medida en que se los incluya en contextos significativos, de manera de crear el juego de correspondencias entre las diferenciaciones formales y sus significados funcionales, estilísticos, simbólicos, económicos, sociológicos, étnicos, etc. (Bromberger 1979).

$\mathrm{El}$ análisis descriptivo constituye un primer paso en este proceso de contextualización, asumiendo la condición de explicitar los criterios a partir de los cuales se aisla un elemento como significativo de manera de exponer los procedimientos y argumentaciones intercontextuales. En el caso de los materiales etnográficos, ellos presentan características particulares referidas al contexto de recolección, que deben ser incorporadas a la hora de construir un modelo de análisis.

RECA, M.M. The object and the construction of meaning in ethnographic collections. Rev. do Museu de Arqueologia e Etnologia, São Paulo, 6: 269-273, 1996.

\begin{abstract}
The conditions for analysis of the material culture and the conceptual marks from which the objects can be read, acquire a singular character in the study of ethnographic collections. Starting from the presentation of methodologic-theoretic aspects related to the considered type of referent and to the enquiry procedures to which the «objects» are submitted by the researcher, we propose several levels of contextualization which make the passage from the material dimension to the symbolic one, which constitute the referential instances of attribution of meaning, to stand out. This process is exemplified in a set of masks which belong to the Ethnography Scientific Departament of the La Plata Museum.
\end{abstract}

UNITERMS: Material culture - Collection - Ethnography - Meaning context - Descriptive study.

\title{
Bibliografia
}

ADAMS, $M$.

1984 Mediatizar nuestro saber. Museum, XXXVI.

BATESON, G.

1976 Pasos hacia una ecología de la mente. Buenos Aires, De. Lolhlé.
BROMBERGER, C.

1979 Technologie et analyse sémantique des objets: pour une sémio technologie. L'Homme, XIX (1): 105-140. 
RECA, M.M. El objeto y la construcción de sentido en colecciones etnográficas. Rev. do Museu de Arqueologia e Etnologia, São Paulo, 6: 269-273, 1996.

CLIFFORD, J.; MARCUS, G.

1991 Retóricas de la Antropología. Madrid, de Jucar. GARDIN, J. C.

1968 Análisis documental y análisis estructural en arqueología. Lévi Strauss (Org.) Estructuralismo y dialéctica. Bs. As., Paidós: 121-128.

GARDIN, J.C. et al.

1975 Essais d'analysis du discours arqueologique. Notes et monographies techniques, 7.

GEERTZ, C.

1978 La interpretación de las culturas. Barcelona, Gedisa.

\section{HODDER, I.}

1988 Interpretación en arqueología. Barcelona, de crítica.

LEVI-STRAUSS, $\mathrm{C}$.

1981 La vía de las máscaras. México, Siglo XXI.

\section{LAHITTE, $\mathrm{H}$.}

1992 Sistemas congnitivos y representación en piezas etnográficas. La Plata, U.N.L.P., cuadernos LARDA, 12 (3).

MATHIEU, J

1987 L'objet et ses contextes. Bulletin d'histoire de la culture materielle, 26 , Canadá

MOLES, A.

1969 Objeto y comunicación. París, Comunications, 13. PALAVECINO, $\mathrm{E}$.

1954 La máscara y la cultura. Bs. As., Ediciones de la Municipalidad de Bs. As.

ROCA, M. et al

1972 El carnaval chiriguano chané. Cuadernos del INA, Bs. As., 8.

TONKIN, E.

1979 Mask and powers. Man, 14 (2) june: 237-248.

Recebido para publicação em 21de novembro de 1996. 